\title{
Elbow torque ellipses: investigation of the mutual influences of rotation, flexion, and extension torques
}

\author{
Fabian Guenzkofer ${ }^{*}$, Heiner Bubb and Klaus Bengler \\ Institute of Ergonomics, Technische Universität München, Boltzmannstr.15, 85747 Garching, Germany
}

\begin{abstract}
Most strength tests are restricted to measuring joint torques only in main directions like flexion or pronation. However, for an advanced strength prediction model, information about maximum possible joint torques in intermediate directions also has to be known. This paper concentrates on obtaining functions for maximum joint torques in arbitrary directions in the elbow. Therefore, isometric joint torque measurements have been conducted with 20 young males applying joint torques in eight different directions in one posture ( 90 degrees elbow flexion, 0 degrees shoulder flexion, neutral forearm rotation). It turns out that the appearance of the resulting shape can be best predicted using ellipse segments. Furthermore, a standardized ellipse could be obtained for the measurement posture. This helps to predict maximum joint torques in any other direction by knowing only one of the main joint torques.
\end{abstract}

Keywords: digital human model, joint torque, biomechanics, elbow, strength

\section{Introduction}

Strength prediction for digital human models presupposes correlations between joint angles and joint torques [27] due to the length - tension relationship of muscles $[6,33]$. Usually joint torque measurements only take place in certain planes and only consider a torque application of one degree of freedom (df) per measurement. Concerning elbow flexion and extension, several authors have examined elbow flexion torques based on different elbow flexion angles $[7,8,10,17,18,20,21,24,29-32]$. As biarticular muscles and muscles which support more than one df are involved, further joint angle variations should be taken into account $[11,23]$. In consequence, some studies also investigated the influence of different forearm rotation angles on elbow flexion torques [9, $10,14,22]$. Only few experiments can be found that took into account all relevant biomechanical aspects and additionally varied shoulder flexion angles [11, 34]. Although most joint torque measurements only consider torque application of one $\mathrm{df}$, real applica- tions often need a simultaneous activation of several degrees of freedom. External forces have to be compensated by internal joint torques pointing in arbitrary directions [28]. However, most hitherto studies have not examined possible coupling effects and therefore existing digital human models disregard them. For example the three-dimensional static strength prediction program (3DSSPP) allows a prediction of flexion / extension joint torques depending on elbow and shoulder flexion angles [5, 26]. As only one $\mathrm{df}$ is considered, pronation and supination as well as possible interaction effects are neglected. Kumar [16] states that "is is unclear how the simplified representation of the upper extremity in these models affects their results". 3DSSPP is also the basis of the static strength prediction tool of the digital human model Jack. Human Builder uses the results from Askew at el. [2] and therefore assumes one elbow flexion joint torque independent of the posture.

Considering biomechanical aspects, interaction effects of flexion/extension and supination/pronation have to be expected. Taking into account participating muscles it is evident that some muscles support

\footnotetext{
* Corresponding author. E-mail: guenzkofer@tum.de, Phone +49 89289 15405, Fax +49 8928915389
} 
joint torque production for both dfs. For example elbow flexion and pronation share the following muscles: $\mathrm{m}$. brachioradialis, $\mathrm{m}$. pronator teres, $\mathrm{m}$. extensor carpi radialis brevis et longus, $\mathrm{m}$. flexor carpi radialis et ulnaris, $m$. flexor digitorum superficialis and m. palmaris longus [23]. Therefore, when simultaneously demanding two dfs of a joint some muscles have to distribute their power. Thus, it is not supposed that the obtained maximum values of single-df- joint torque measurements can be vectorially added in order to get torque predictions for intermediate directions.

Similar to force ellipsoids for maximum endeffector forces [35] due to Schaefer [25], maximum joint torques in directions apart from the measurement axes are supposed to shape so called Mpotatoes.

However, the real appearance has not been validated up to now. Figure 1 depicts the problem using exemplarily an intermediate joint torque between supination and flexion. Measurements lead to maximum joint torques for flexion and supination but the amount of an intermediate joint torque in a given direction is unknown.

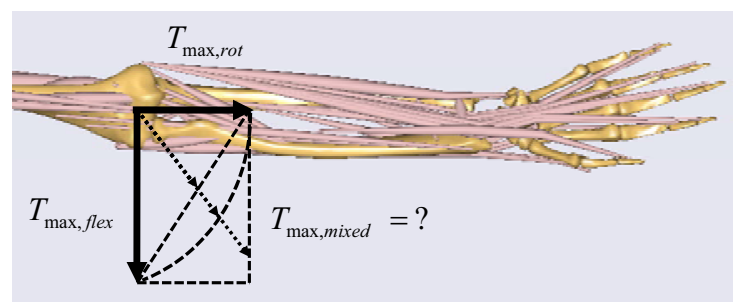

Figure 1: Illustration of maximum elbow flexion and supination joint torque in a right arm model. Different possibilities for the prediction of an intermediate joint torque are given. The arm model originates from AnyBody (AnyBody Technology, Aalborg, Denmark)

Some authors have already investigated the influence of supination and pronation torques on maximum flexion torque $[3,13]$.

Bechtel et al. [3] have added 50\% supination maximum voluntary contraction (MVC) to flexion MVC for five different elbow flexion angles. They found out that maximum flexion torques were hereby reduced. That means the maximal flexion torque depends on additional rotation torques. Overlaying pronation and flexion as well as maximal pronation and supination torques have not been investigated. Jamison et al. [13] measured maximum elbow flexion torques while applying seven different supination and pronation torque levels. Again, they have not modeled the complete dependence between flexion, extension, supination, and extension.

In sum, all previous studies lack the course from maximum rotation to maximum flexion torques. The whole torque shape has not been investigated so far. Therefore the aim of this study is to investigate maximum intermediate joint torques consisting of a simultaneous activation of flexion/extension and pronation/supination torques and their relation to joint torques in main directions.

\section{Material and methods}

\subsection{Subjects}

Twenty young, male subjects who indicated themselves as healthy and free of orthopedic and neurological disorders, volunteered to take part in this study. (see table 1). All subjects gave their written consent after having been informed about the purpose of the study and its procedures.

Table 1. Mean and standard deviation of age, height and weight of participants in each group

\begin{tabular}{ll}
\hline Age & $23,9 \pm 1,8$ years \\
Height & $183,3 \pm 6,7 \mathrm{~cm}$ \\
Weight & $79,5 \pm 9,2 \mathrm{~kg}$ \\
\hline
\end{tabular}

\subsection{Apparatus}

A self designed device with several adjustment possibilities was used for measuring the elbow joint torques (see figure 2). One torque sensor registers forearm rotation torques and another flexion and extension torques (burster präzisionsmesstechnik gmbh \& co kg, Gernsbach, Germany).

The whole device is adjustable in height to compensate for different sitting heights of the seated subjects. Shoulder bolsters serve for stabilizing the subject. The elbow torque sensor and upper arm bolsters can be adjusted in length and height in order to align the anatomical joint axis and the measurement axis. Adjustable forearm bolsters ensure comfortable force application for different forearm lengths. Forearm rotation torques were registered via a handle that was adjustable in length. The torque sensor read-out was conducted using LabVIEW 8.5 (National Instruments Corporation, Austin, USA). 


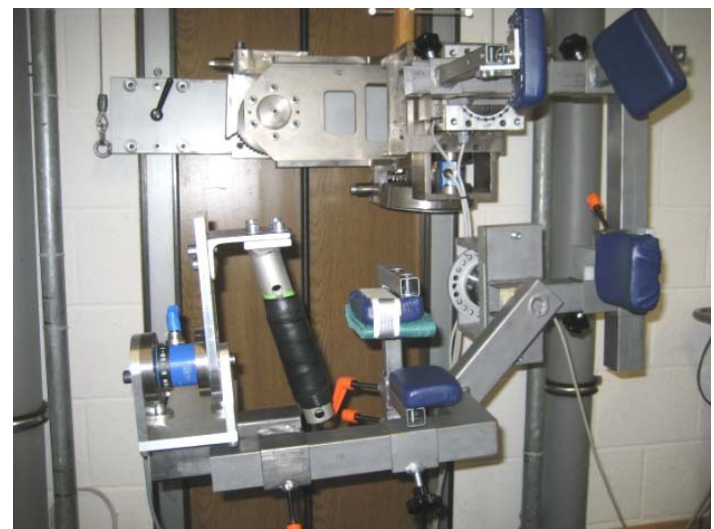

Figure 2: Elbow joint torque measuring device

\subsection{Experimental design}

In order to obtain information about maximum possible joint torques in different orientations, eight directions were determined for one measurement posture. Apart from flexion, extension, supination and pronation as the main directions, intermediate directions between two main directions were also of interest (see figure 3 and table 2).

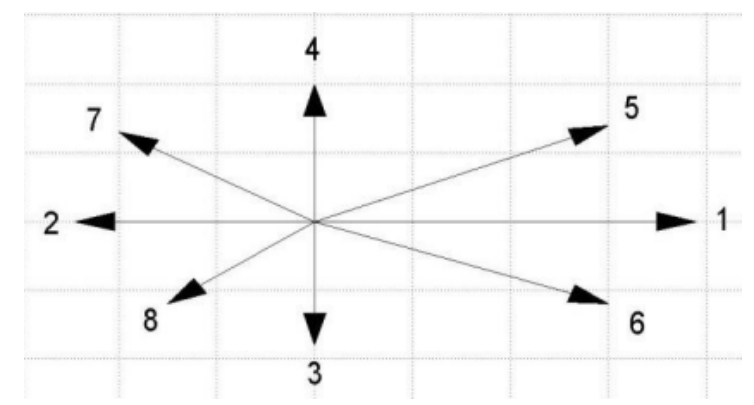

Figure 3: Vector presentation of required joint torque directions

Table 2

Used measurement positions $(\mathrm{F}=$ Flexion, $\mathrm{E}=$ Extension, $\mathrm{P}=$ Pronation, $\mathrm{S}=$ Supination)

\begin{tabular}{|c|l|}
\hline Position & \multicolumn{1}{|c|}{ Task (maximum joint torques) } \\
\hline 1 & elbow flexion \\
\hline 2 & elbow extension \\
\hline 3 & elbow pronation \\
\hline 4 & Elbow supination \\
\hline 5 & Combination of flexion and supination \\
\hline 6 & Combination of flexion and pronation \\
\hline 7 & Combination of extension and supination \\
\hline 8 & Combination of extension and pronation \\
\hline
\end{tabular}

That means that the subjects had to simultaneously apply a maximum flexion and a maximum forearm rotation torque. As the resulting direction is impossible to influence, the only requirement was to apply a simultaneous maximum torque pair. Using these eight positions makes it possible to approximate the geometric form of the resulting torque body.

In order to keep a reasonable measurement effort, only one posture was examined. The chosen posture consisted of shoulder flexion 0 degrees (arm hanging loose), elbow flexion 90 degrees, and a neutral forearm rotation. This posture turned out to lead to the highest joint torques in further studies [11].

For each measurement direction, three trials were performed to ensure that the true maximum was obtained. All in all, this resulted in 24 measurements in total.

To avoid practice and fatigue effects, the order of measurements was randomized for each subject.

Isometric joint torque measurements were performed using the plateau-method, in which the subjects build up their maximum force in the first second and maintain the force for four seconds [15]. The average value over a three second interval during the last four seconds matches the maximum value. In between two trials, the subjects had a rest period of two minutes.

Throughout the experiment the subjects did not receive any kind of external encouragement, neither verbal encouragement nor visual feedback $[4,19]$.

As the experiments were isometric, no motion occurred during the trials. It was therefore very hard for subjects to imagine what kind of motion the applied intermediate torques would yield. Consequently, special warm-up exercises were designed that helped learning how to simulate a combined torque application in a static condition. This was realized using an unladen barbell. An exercise exemplarily consisted of starting an elbow flexion with an extended arm and a neutral forearm position. While flexing the elbow, the forearm had to be supinated, so that the flexion ended with a full supination. All four combinations (see table 2) were realized in warm-up exercises.

\subsection{Procedure}

In the beginning the subjects were instructed on the experimental procedure and familiarized with the measuring device. Afterwards, they performed warmup exercises that additionally served to familiarize with the requested "motions" during the trials. Then the measuring device was adjusted to their anthro- 
pometric dimensions. The elbow flexion axis was approximated by palpating the medial and lateral humeral epicondyles. Thereupon the subjects had the possibility to perform training trials to get used to the measurement positions and the strategy to optimally apply the maximum possible force. For each task the subject was instructed to build up a maximum force within one second and to maintain it for four seconds. Afterwards, a two minutes countdown defined the minimum rest period before the subsequent trial was started. After performing the maximum torque measurements the subjects had to relax their arms in the device and the resulting torque was measured for each measurement posture. Thus, the torque induced by the body segment weight was recorded in order to take into account gravitational influences.

\subsection{Data analysis}

For the main direction, only the highest value of the three repetitions per trial was further processed [15]. In contrast, for the intermediate joint torques all values were taken into account as they were differently oriented in most cases. Net joint torque vectors were visualized in Matlab R2010b (Mathworks, Inc., Natick, MA, USA) for each subject. Using a stepwise approach for each quadrant, a quarter of an ellipse was calculated and plotted (see figure 4).

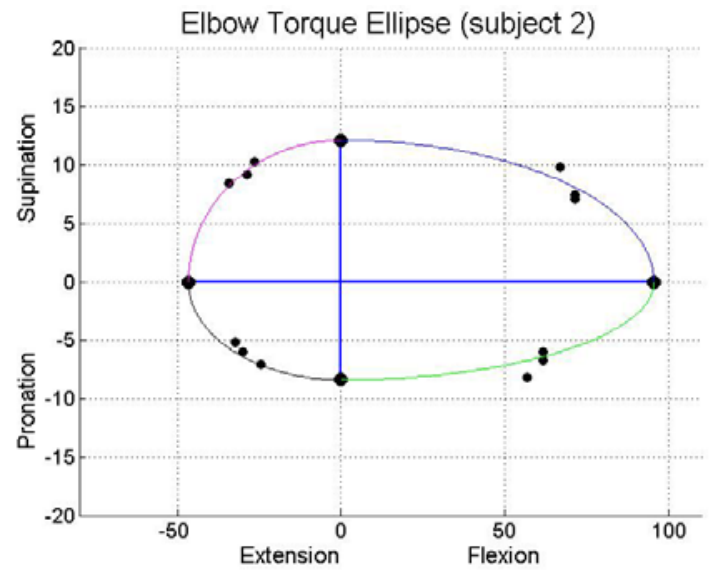

Figure 4: Representative illustration of joint torques in main and intermediate directions for one subject. Torque is given in [Nm].

In order to compare the quality of using an ellipse for a prediction model additionally a rhombus and rectangle were added for each quadrant (cf. figure 1). Then a directional vector was calculated for each intermediate joint torque (see figure 5). Consequently, the intersections between this vector and the three models (ellipse, rhombus, rectangle) were determined. These intersections represent the joint torque predictions in the respective directions (purple circles in figure 5). For each model, the resulting distances between joint torque predictions and the actually occurred joint torques were calculated. Subsequently, for each quadrant the distances were averaged over the three intermediate directions for each model.

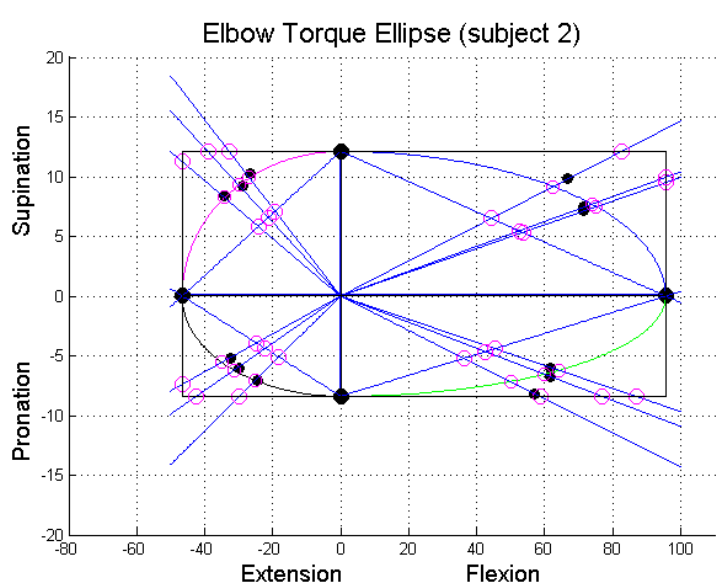

Figure 5: Depiction of three model approaches (ellipse, rhombus, rectangle) for a joint torque prediction in given directions. Torque is given in $[\mathrm{Nm}]$.

Finally, a repeated-measures ANOVA was conducted using model type and quadrant as withinsubject variables. All statistical analyses were performed in the SPSS statistical package, release 18 (SPSS, Inc., Chicago, IL, USA). The level of statistical significance was set at $\mathrm{p}<.05$.

\section{Results}

\subsection{Comparison of theoretical models}

Figure 6 shows the obtained differences for the three models (ellipse, rhombus, and rectangle) separated for each quadrant averaged over all subjects. 


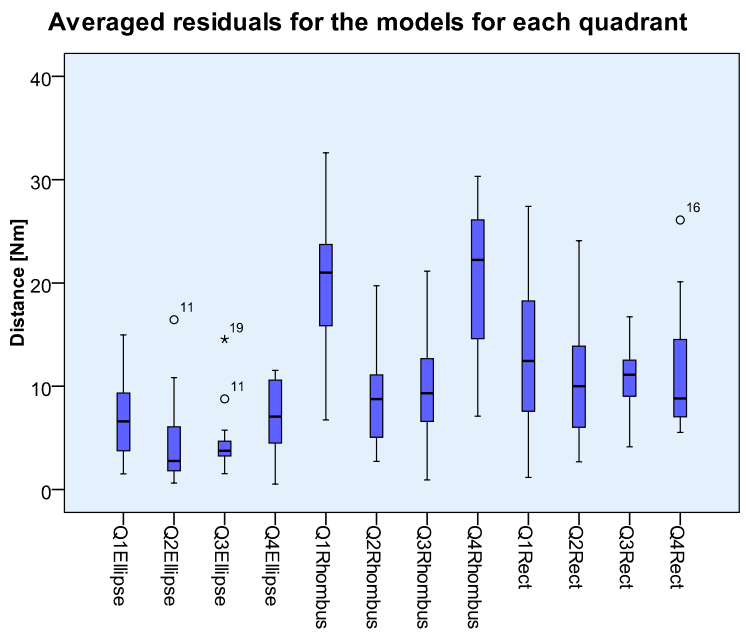

Figure 6: Comparison of the models for each quadrant

It is evident that the ellipse approach leads to the lowest deviations between model and empirical values for all quadrants. Especially for the rhombus approach, a clear dependence on the quadrant is revealed. For a better global comparison, the three models have been averaged over the four quadrants and plotted with each other in figure 7.

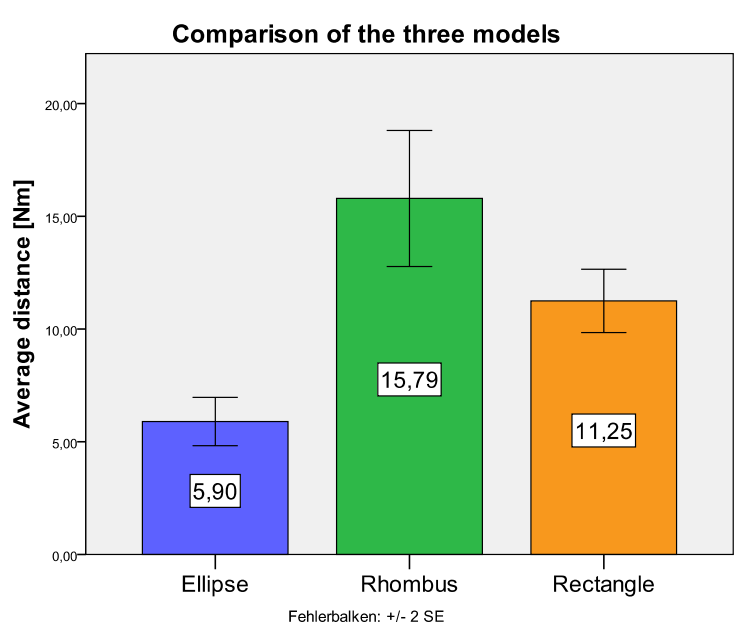

Figure 7: Averaged residuals for the models

Again, it is clearly visible that using an ellipse results in the lowest residuals. After a distinct gap follow the second best approach rectangle and the worst rhombus.
A repeated measures ANOVA serves to support the visually evident results of figure 7 .

Mauchly's test indicated that the assumption of sphericity had been violated for quadrant, $\chi^{2}(5)=$ $15.01, \mathrm{p}<.05$, as well as for the interaction quadrant*model, $\chi^{2}(20)=54.137, \mathrm{p}<.001$. Therefore degrees of freedom were corrected using the estimates of Greenhouse-Geisser $(=.64$ and $=.54)$. It turned out that the models are significantly different, $\mathrm{F}(2,36)=41.19, \mathrm{p}<.001$. Besides, the residuals significantly depend on the quadrant, $F(1.91,34.31)$ $=19.14, \mathrm{p}<0.001$. Finally, the amount of deviations for each model depends on the quadrant, $F(3.25$, $58.61)=6.65, \mathrm{p}<.001$. Bonferroni post hoc tests indicate that all models are significantly different. Further Bonferroni post hoc tests show that all quadrants are significantly different apart from quadrant one and four, and quadrant two and three.

\subsection{Standardized torque ellipse}

For all subjects, joint torques have been measured in the main directions. Therefore, a standardized ellipse for an average young male could be modeled. Therefore the means, standard deviations, minimum, and maximum values were calculated for each direction (see table 3).

Table 3

Mean, standard deviation, minimum, and maximum joint torque $[\mathrm{Nm}]$ for each direction averaged over all subjects

\begin{tabular}{lcccc}
\hline & Flexion & Extension & Pronation & Supination \\
\hline Mean & 78,57 & 49,16 & 8,39 & 10,11 \\
Std & 11,07 & 11,46 & 1,64 & 2,22 \\
Min & 58,85 & 73,59 & 11,62 & 6,12 \\
Max & 101,25 & 26,19 & 5,66 & 13,82
\end{tabular}

The mean values can now be used to design a standard torque ellipse predicting the maximum elbow joint torques for the chosen measurement posture.

Additionally, the dataset paves the way for another possibility of reducing measurement effort for future experiments. If the ratios of the four joint torques in the main directions were stable, it would suffice to only measure elbow flexion for one posture in order to model the whole ellipse for the chosen posture. Therefore the means, standard deviations, minimum and maximum value of the ratios were calculated (see table 4 and figure 8 ). 
Table 4

Mean, standard deviation, minimum, and maximum joint torque ratios averaged over all subjects $(\mathrm{F}=$ Flexion, $\mathrm{E}=$ Extension, $\mathrm{P}=$ Pronation, $\mathrm{S}=$ Supination)

\begin{tabular}{lcccc}
\hline & $\mathbf{F} / \mathbf{E}$ & $\mathbf{P} / \mathbf{S}$ & $\mathbf{F} / \mathbf{S}$ & $\mathbf{E} / \mathbf{S}$ \\
\hline Mean & 1,66 & 0,85 & 8,02 & 5,01 \\
Std & 0,32 & 0,16 & 1,41 & 1,26 \\
Min & 1,20 & 0,60 & 6,08 & 3,16 \\
Max & 2,48 & 1,14 & 11,09 & 7,48 \\
\hline
\end{tabular}

Averaged Ratios of different measurement directions

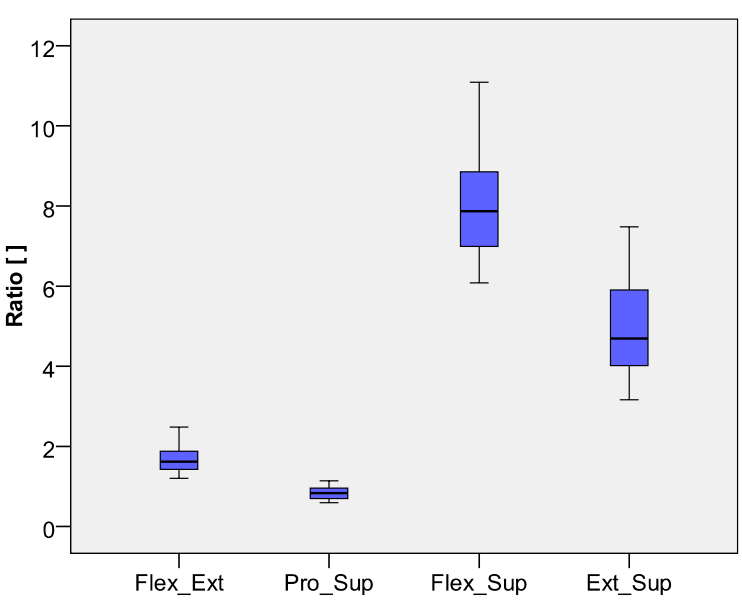

Figure 8: Averaged ratios between measurement directions

In order to estimate the quality of the ratios, based on elbow flexion the torques of the other directions were calculated using the information of table 4 . The predicted values were then compared with the real values for each subject. Finally, the resulting differences were averaged over all subjects (table 5).

Table 5.

Mean, standard deviation, minimum, and maximum joint torque ratios averaged over all subjects $(\mathrm{F}=$ Flexion, $\mathrm{E}=$ Extension, $\mathrm{P}=$ Pronation, $\mathrm{S}=$ Supination)

\begin{tabular}{cccc}
\hline & Extension & Pronation & Supination \\
\hline Mean & 1,73 & 0,05 & 0,31 \\
\hline
\end{tabular}

That means for example when predicting elbow extension based on the elbow flexion torque the deviation will be around $1,73 \mathrm{Nm}$ on average. However, it has to be noted that for only few subjects high deviations would be obtained (see minimum and maximum ratios in table 4 ).

\section{Discussion}

The results of the present study are in line with those of Jamison et al. [13] and Bechtel et al. [3]. Also, in this study, flexion joint torques decreased when simultaneously applying supination or pronation joint torques.

The population of participants used in this study is comparable to those used in other studies. Amis et al. [1980] as well as Winters and Kleweno [34] obtained approximately $70 \mathrm{Nm}$ for elbow flexion in the same posture. Even Askew et al. [2], who performed measurements using 50 males, obtained $71 \mathrm{Nm}$ for male subjects.

Statistical analysis showed that joint torques in intermediate directions can best be predicted using an ellipse. However, it was shown that model accuracy depends on the quadrant. For example the rhombus model was quite good for quadrant two and three, but globally the worst for quadrants one and four (see figure).

The dependency of maximum flexion torque on supination and pronation torque makes sense considering that some muscles are shared, such as: as $\mathrm{m}$. biceps brachii, $\mathrm{m}$. pronator teres, and $\mathrm{m}$. brachioradialis. Therefore, when additionally serving for another df less capacity stays for the actual df.

However, for elbow extension no muscular reason in terms of shared muscles can be found. Nevertheless a distinct influence on supination and pronation torques was obtained. In accordance to Jamison et al. [13] it is assumed that "the second df task affects some aspect of joint function that causes the CNS to reduce $\mathrm{F}$ torque capacity".

Besides establishing the ellipse model, it was shown that it is possible to construct an individual ellipse based on only one measurement. Therefore, it is enough to measure only elbow flexion in future experiments and obtain the rest of the ellipse by the obtained ratios. This could lead to some deviations, but the accuracy should be sufficient for practical reasons as high variances are inherent to strength measurements anyway [15].

Nevertheless, this study has some limitations. It was only conducted with young male subjects. Therefore it is debatable whether the results are also applicable for older subjects and for female subjects. Guenzkofer et al. [11, 12] have shown that at least for the shape of the joint torque - joint angle functions significant differences could be found. Furthermore, the subjects performed 24 measurements, but only in one posture. It is assumed that the shape 
of the ellipses, above all in terms of the appearance of the single quadrants, will change for other joint angles. It is true that using the stepwise-approach all other positions can be modeled as well, but the ratios of table 4 will no longer be valid.

\section{Conclusions}

In this paper it was shown that joint torques in intermediate directions - that means between flexion / extension and pronation / supination - can be well predicted using an ellipse. Furthermore for the used posture of 90 degrees elbow flexion and 0 degrees shoulder flexion a standardized ellipse could be obtained for young males. That is to say by only knowing one maximum joint torque, the joint torques in other directions can be predicted quite well. These findings can now be implemented in digital human models in order to improve strength prediction. Instead of only using force information in one plane like in the 3DSSPP, spatial force information can then be taken into account.

\section{Acknowledgements}

The authors thank Mathias Hoellige for enthusiastically supporting the practical test conduction. The research leading to these results has received funding from the European Community's Seventh Framework Programme (FP7/2007-2013) under grant agreement no 218525. The authors gratefully acknowledge the support of the TUM Graduate School at Technische Universität München, Germany.

\section{References}

[1] Amis, A. A., Dowson, D., \& Wright, V. (1980). Elbow joint force predictions for some strenuous isometric actions. Journal of Biomechanics, 13(9), 765-775.

[2] Askew LJ, An KN, Morrey BF, \& Chao EY. (1987). Isometric elbow strength in normal individuals. Clinical orthopaedics and related research, (222), 261-266.

[3] Bechtel, R., \& Caldwell, G. E. (1994). The influence of task and angle on torque production and muscle activity at the elbow. Journal of Electromyography and Kinesiology, 4(4), 195-204.

[4] Brown, L. E., \& Weir, J. P. (2001). ASEP Procedures recommendation 1: Accurate Assessment of muscular strength and power. Journal of Exercise Physiology (online), (Volume 4 Number 3).

[5] Chaffin, D. B. (1998). Prediction of Population Strength. Proceedings of the SAE DHMC (Digital Human Modeling Conference), (SAE 981307).
[6] Chaffin, D. B., Andersson, G., \& Martin, B. J. (2006). Occupational biomechanics (4th ed.). Hoboken N.J.: WileyInterscience.

[7] Clarke, H. H., Elkins, E. C., Martin, G. M., \& Wakim, K. G. (1950). Application of muscle power to movements of the joints. Arch. Phys. Med. Rehabil., (31), 81-89.

[8] Doss, W. S., \& Karpovich, P. V. (1965). A comparison of concentric, eccentric, and isometric strength of elbow flexors. Journal of Applied Physiology, 20(2), 351-353.

[9] Downer AH. (1953). Strength of the elbow flexor muscles. The Physical therapy review, 33(2), 68-70.

[10]Elkins EC, Leden UM, \& Wakim KG. (1951). Objective recording of the strength of normal muscles. Archives of physical medicine and rehabilitation, 32(10), 639-647.

[11] Guenzkofer, F., Engstler, F., Bubb, H., \& Bengler, K. (2011) Isometric elbow flexion and extension joint torque measurements considering biomechanical aspects. In Proceedings of the First International Symposium on Digital Human Modeling. Lyon.

[12] Guenzkofer, F., Engstler, F., Bubb, H., \& Bengler, K. (2011) Joint Torque Modeling of Knee Extension and Flexion. In Proceedings of the 14th International Conference on HumanComputer Interaction. Orlando, Florida, USA.

[13] Jamison, J. C., \& Caldwell, G. E. (1993). Muscle Synergies and isometric torque production - influence of supination and pronation level on elbow. Journal of Neurophysiology, 70(3), 947-960.

[14]Jorgensen, K., \& Bankov, S. (1971). Maximum strength of elbow flexors with pronated and supinated forearm. E. Jokl (Ed.) Biomechanics II Basel, Karger, (174-180).

[15]Kumar, S. (2004). Muscle strength. Boca Raton: CRC Press.

[16]Kumar, S. (2008). Biomechanics in ergonomics (2nd). Boca Raton: CRC Press.

[17]Leedham JS, \& Dowling JJ. (1995). Force-length, torqueangle and EMG-joint angle relationships of the human in vivo biceps brachii. European journal of applied physiology and occupational physiology, 70(5), 421-426.

[18]Linnamo V, Strojnik V, \& Komi PV. (2006). Maximal force during eccentric and isometric actions at different elbow angles. European journal of applied physiology, 96(6), 672-678. doi:10.1007/s00421-005-0129-x

[19] Mital, A., \& Kumar, S. (1998). Human muscle strength definitions, measurement, and usage: Part I - Guidelines for the practitioner. International Journal of Industrial Ergonomics, 22(1-2), 101-121.

[20] Osternig LR, Bates BT, \& James ST. (1977). Isokinetic and isometric torque force relationships. Archives of physical medicine and rehabilitation, 58(6), 254-257.

[21] Petrofsky JS, \& Phillips CA. (1980). The effect of elbow angle on the isometric strength and endurance of the elbow flexors in men and women. Journal of human ergology, 9(2), $125-131$.

[22] Provins KA, \& Salter N. (1955). Maximum torque exerted about the elbow joint. Journal of applied physiology, 7(4), 393-398.

[23] Ramsay JW, Hunter BV, \& Gonzalez RV. (2009). Muscle moment arm and normalized moment contributions as reference data for musculoskeletal elbow and wrist joint models. Journal of biomechanics, 42(4), 463-473. doi:10.1016/j.jbiomech.2008.11.035

[24] Sato, M., \& Sakai, Y. (1968). Isometric and eccentric strength of elbow flexors and extensors. J. Anthropol. Soc. Nippon, (76), 183-190.

[25] Schaefer, P., Rudolph, H., \& Schwarz, W. (2000). Digital Man Models and Physical Strength - A New Approach in 
Strength Simulation. Proceedings of the SAE DHMC (Digital Human Modeling Conference), (SAE 2000-01-2168).

[26] Schanne, F. (1972). A Three Dimensional Hand Force Capability Force Model for the Seated Operator: Unpublished doctoral dissertation.

[27] Schwarz, W. (1997). 3D-Video-Belastungsanalyse: Ein neuer Ansatz zur Kraft- und Haltungsanalyse (Als Ms. gedr.). Fortschritt-Berichte VDIReihe 17, Biotechnik/Medizintechnik: Vol. 166. Düsseldorf: VDI-Verl.

[28] Seitz, T., Recluta, D., Zimmermann, D., \& Wirsching, H.-J. (2005). FOCOPP - An approach for a human posture prediction model using internal/external forces and discomfort. Proceedings of the SAE DHMC (Digital Human Modeling Conference), (SAE 2005-01-2694).

[29] Singh M, \& Karpovich PV. (1968). Strength of forearm flexors and extensors in men and women. Journal of applied physiology, 25(2), 177-180.

[30] Tsunoda N, O'Hagan F, Sale DG, \& MacDougall JD. (1993). Elbow flexion strength curves in untrained men and women and male bodybuilders. European journal of applied physiology and occupational physiology, 66(3), 235-239. [31]van Zuylen EJ, van Velzen A, \& Denier van der Gon JJ. (1988). A biomechanical model for flexion torques of human arm muscles as a function of elbow angle. Journal of biomechanics, 21(3), 183-190.

[32] Williams, M., \& Stutzmann, L. (1959). Strength variation through the range of motion. Phys. Ther., (39), 145-152.

[33] Winter, D. A. (2005). Biomechanics and motor control of human movement (3. ed.). Hoboken, NJ: Wiley.

[34]Winters JM, \& Kleweno DG. (1993). Effect of initial upperlimb alignment on muscle contributions to isometric strength curves. Journal of biomechanics, 26(2), 143-153.

[35]Zatsiorsky, V. M. (2002). Kinetics of human motion. Champaign, Ill.: Human Kinetics. Retrieved from 